\title{
In Situ Synthesis of Magnetite Nanoparticles in Carrageenan Gels
}

\author{
Ana L. Daniel-da-Silva, ${ }^{*}, \dagger$ T. Trindade, ${ }^{\dagger}$ Brian J. Goodfellow, ${ }^{\dagger}$ Benilde F. O. Costa, ${ }^{\ddagger}$ \\ Rui N. Correia, ${ }^{\S}$ and Ana M. Gil ${ }^{\dagger}$ \\ Department of Chemistry, CICECO, and Department of Ceramics and Glass Engineering, University of \\ Aveiro, 3810-193 Aveiro, Portugal, and Department of Physics, University of Coimbra, \\ 3004-516 Coimbra, Portugal
}

Received January 25, 2007; Revised Manuscript Received June 6, 2007

\begin{abstract}
Magnetite nanoparticles have been successfully synthesized in the presence of carrageenan polysaccharides using an in situ coprecipitation method. Iron coordination to the sulfate groups of the polysaccharide was confirmed by FTIR. The polysaccharide type $(\kappa, \iota$, or $\lambda)$ and concentration have been varied and their effects on particle morphology and chemical stability of the resultant nanocomposite investigated. The presence of carrageenan induces the formation of smaller particles, compared to those formed in the absence of polymer, and their average size depends on the nature and concentration of the polysaccharide used. The chemical stability of magnetite nanoparticles toward oxidation was also seen to depend on biopolymer type with magnetite formed in $l$-carrageenan showing the highest chemical stability. A general tendency toward lower stability is observed as the polysaccharide concentration is increased. It is suggested that magnetite chemical stability in the carrageenan composites is determined by a fine balance between particle size and gel strength, the latter determining oxygen diffusion rates through the medium.
\end{abstract}

\section{Introduction}

Over the past few years, synthetic control of nanoparticle morphology and composition has been achieved using a variety of wet chemical methods. ${ }^{1-9}$ Polymers have been extensively used to control particle size, acting as stabilizers and preventing the spontaneous agglomeration of nanoparticles, allowing functional nanomaterials to be produced and subsequently used in pharmaceutical and biomedical products. ${ }^{10-12}$ Biopolymers are good candidates for use in the biomedical field, as they are nontoxic and confer biocompatibility to the resulting nanocomposite material. However, at present, there is a lack of information on the mechanisms of formation of nanoparticles in the presence of these biopolymers.

Many successful examples of the use of polysaccharides for controlled syntheses of iron oxide nanoparticles can be found. Due to their low toxicity, magnetic iron oxides, such as magnetite $\left(\mathrm{Fe}_{3} \mathrm{O}_{4}\right)$ and maghemite $\left(\gamma-\mathrm{Fe}_{2} \mathrm{O}_{3}\right)$, have long been recognized as potential components in biomaterials. Indeed, an increasing number of medical applications, including drug targeting, bioseparation processes, cancer thermotherapy, and use as contrast agents in magnetic resonance imaging (MRI), have been found for iron oxide nanoparticles. ${ }^{11,13,14}$ Conventional chemical synthesis of $\mathrm{Fe}_{3} \mathrm{O}_{4}$ and $\gamma-\mathrm{Fe}_{2} \mathrm{O}_{3}$ powders involves the coprecipitation of a mixture of aqueous ferrous and ferric salts. ${ }^{15}$ Previous studies have shown that some polysaccharides have the ability to interact with iron complexes, preventing the precipitation of iron hydroxide and enabling nanoparticle synthesis to occur under controlled conditions. For example, several workers have used a coprecipitation process in the presence of dextran. ${ }^{9,16-18}$ Other biopolymers used include cyclodextrin, ${ }^{19}$ alginate, ${ }^{20,21}$ chitosan, ${ }^{10,21}$ starch-based coatings, ${ }^{12,21,22}$ and $\kappa$-carrageenan. ${ }^{23,24}$ Furthermore, specific studies have been carried out on the interaction of $\kappa$-carrageenan with several transition-metal oxides and a structural model has been proposed for the biomineralization of iron oxides in the presence of this polysaccharide..$^{23,25}$ As a result, the use of $\kappa$-carrageenan

* Corresponding author. Tel.: +351234 370 261.Fax.: +351234 425 300. E-mail: ana.silva@dq.ua.pt.

$\dagger$ Department of Chemistry, CICECO, University of Aveiro.

Department of Physics, University of Coimbra.

$\S$ Department of Ceramics and Glass Engineering, University of Aveiro.
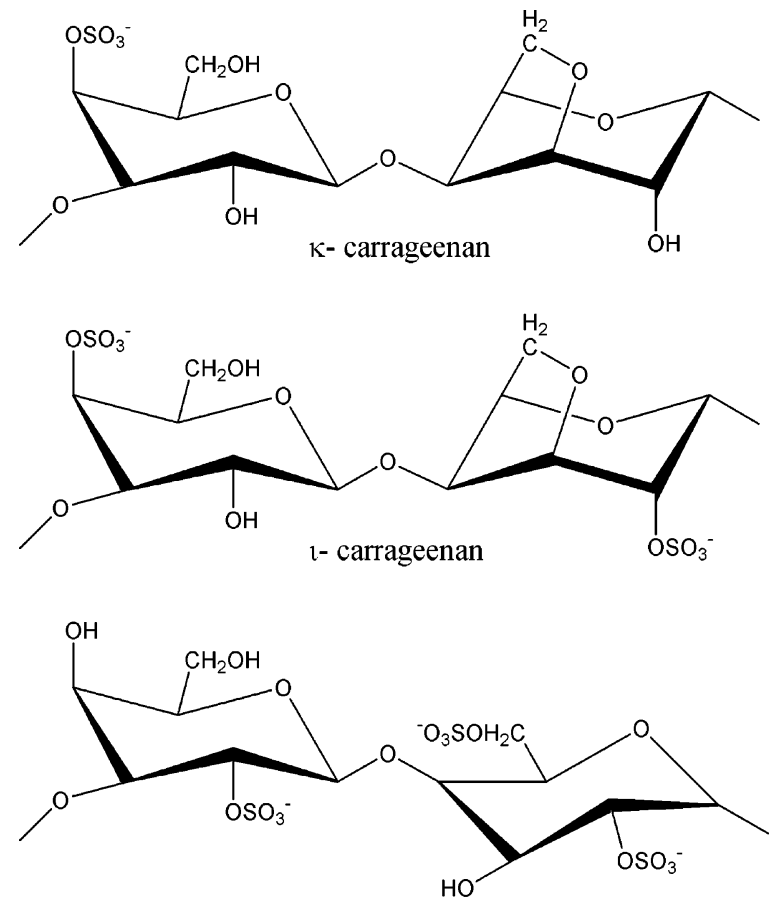

$\lambda$ - carrageenan

Figure 1. Disaccharide repeat units of $\kappa^{-}, \iota^{-}$, and $\lambda$-carrageenan.

as coating for magnetic particles has been suggested in order to induce the formation of a self-assembled nanoreactor within which iron oxides nanoparticles are stabilized. ${ }^{23}$

In this work, $\kappa^{-}, \lambda$-, and $\iota$-carrageenan have been used as a media for the synthesis of magnetite nanoparticles, and the morphology and chemical stability of the resulting nanocomposites toward oxidation have been investigated. These biopolymers comprise a group of linear sulfated polysaccharides extracted from red seaweeds composed of a repeating disaccharide unit that differs in sulfate content/position as well as in the presence of an anhydrous bridge on one of the monomers (Figure 1). Carrageenans form thermoreversible gels, often 
Table 1. Synthesis Conditions of Magnetic Particles

\begin{tabular}{cccc}
\hline $\begin{array}{c}\text { sample } \\
\text { designation }\end{array}$ & carrageenan & $\begin{array}{c}C_{\text {carrageenan }} \\
(\mathrm{g} / \mathrm{L})\end{array}$ & $\begin{array}{c}\text { alkali } \\
\text { solution } \\
(1 \mathrm{M})\end{array}$ \\
\hline $0 \mathrm{~K}$ & & 0 & $\mathrm{KOH}$ \\
$\kappa 1 \mathrm{~K}$ & $\kappa$ & 1 & $\mathrm{KOH}$ \\
$\kappa 5 \mathrm{~K}$ & $\kappa$ & 5 & $\mathrm{KOH}$ \\
$\kappa 10 \mathrm{~K}$ & $\kappa$ & 10 & $\mathrm{KOH}$ \\
$\kappa 20 \mathrm{~K}$ & $\kappa$ & 20 & $\mathrm{KOH}$ \\
$\kappa 40 \mathrm{~K}$ & $\kappa$ & 40 & $\mathrm{KOH}$ \\
$\kappa 20 \mathrm{Na}$ & $\kappa$ & 20 & $\mathrm{NaOH}$ \\
$\kappa 20 \mathrm{Cs}$ & $\kappa$ & 20 & $\mathrm{CsOH}$ \\
$\iota 5 \mathrm{~K}$ & $\iota$ & 5 & $\mathrm{KOH}$ \\
$\iota 10 \mathrm{~K}$ & $\iota$ & 10 & $\mathrm{KOH}$ \\
$\iota 20 \mathrm{~K}$ & $\iota$ & 20 & $\mathrm{KOH}$ \\
$\iota 40 \mathrm{~K}$ & $\iota$ & 40 & $\mathrm{KOH}$ \\
$\lambda 5 \mathrm{~K}$ & $\lambda$ & 5 & $\mathrm{KOH}$ \\
$\lambda 10 \mathrm{~K}$ & $\lambda$ & 10 & $\mathrm{KOH}$ \\
$\lambda 20 \mathrm{~K}$ & $\lambda$ & 20 & $\mathrm{KOH}$ \\
$\lambda 40 \mathrm{~K}$ & $\lambda$ & 40 & $\mathrm{KOH}$ \\
\hline
\end{tabular}

stabilized by alkali-metal cations and, due to their nontoxicity and water solubility, have been widely used as gelling agents in the food and pharmaceutical industries. ${ }^{26}$ This study aims to investigate the role of carrageenan type and concentration and nature of alkali-metal cation in the formation and characteristics (size and chemical stability) of magnetite nanoparticles. These systems are of particular interest, not only because reaction conditions may be tailored for size and stability of the resulting iron oxide but also because controlled release of magnetic particles may be envisaged.

\section{Experimental Section}

2.1. Materials. $\kappa$-Carrageenan (batch no. 442129), $\iota$-carrageenan (batch no. 406890), and $\lambda$-carrageenan (batch no. 1260935) were obtained from Fluka Chemie and used as received. Ferric chloride $\left(\mathrm{FeCl}_{3}\right)\left(>98 \%\right.$, Merck), ferrous sulfate heptahydrate $\left(\mathrm{FeSO}_{4} \cdot 7 \mathrm{H}_{2} \mathrm{O}\right)$ $(>99 \%$, Panreac), sodium hydroxide $(\mathrm{NaOH})(>98 \%$, Akzo Nobel), potassium hydroxide $(\mathrm{KOH})(>86 \%$, Akzo Nobel), and cesium hydroxide $(\mathrm{CsOH})(>95 \%$, Fluka Chemie) were used as received without further purification.

2.2. Iron Oxide Nanoparticle Synthesis. The magnetic nanoparticles were produced by in situ chemical coprecipitation. In a typical procedure, $2 \mathrm{~mL}$ of acidified iron(III) chloride $(0.2 \mathrm{M})$, freshly prepared in deoxygenated water and $65 \mathrm{mg}$ of iron(II) sulfate heptahydrate were added to $20 \mathrm{~mL}$ of deoxygenated carrageenan solution at $60{ }^{\circ} \mathrm{C}$, under a nitrogen atmosphere. Ferrous ion was added in slight excess of the magnetite stoicheometric molar ratio $\left(\mathrm{Fe}^{3+} / \mathrm{Fe}^{2+}=2: 1\right)$ in order to account for the possible oxidation of $\mathrm{Fe}^{2+}$. Then $2.2 \mathrm{~mL}$ of either $1 \mathrm{M}$ sodium, potassium, or cesium hydroxide was added immediately or after $10 \mathrm{~min}$ stirring giving a final $\mathrm{pH}$ of above 10 . The mixture immediately turns black, which, together with the observed magnetic properties, indicates the presence of magnetite. Counterion type, polysaccharide type, and polysaccharide concentration were varied as shown in Table 1.

2.3. Material Characterization. Characterization of the polysaccharide/magnetite nanocomposites by X-ray diffraction analysis (XRD), Fourier transform infrared spectroscopy (FTIR), and Mössbauer spectroscopy was carried out on freeze-dried samples of the suspension obtained by magnetic separation from the dilute solutions. The crystallite phase was identified by recording X-ray powder diffraction patterns of the freeze-dried samples using a Rigaku Geigerflex Dmax-C X-ray difractometer equipped with a $\mathrm{Cu} \mathrm{K} \alpha$ monochromatic radiation source. FTIR spectra (128 scans at a resolution of $4 \mathrm{~cm}^{-1}$ ) were collected using a Mattson 7000 spectrometer coupled to a horizontal attenuated total reflectance (ATR) cell. Mössbauer measurements were performed on the freeze-dried samples, at room temperature, using a constantacceleration spectrometer in transmission geometry with a ${ }^{57} \mathrm{Co} / \mathrm{Rh}$ source. The spectral fitting was carried out using a set of Lorentzian lines determined by least-squares. In addition, freeze-dried gels without magnetic separation of the nanoparticles were also analyzed by Mössbauer spectroscopy. These samples contain higher biopolymer concentrations and nonmagnetic iron oxide byproducts of the magnetite synthesis may be present.

For characterization by transmission electron microscopy (TEM) and visible-near-infrared spectroscopy (vis-NIR), nanoparticle samples were treated in the following manner. For TEM analysis, samples were prepared by evaporating dilute suspensions of the magnetically collected particles on a copper grid coated with an amorphous carbon film. In the case of the particles prepared in the absence of the biopolymer, oleic acid was added, and the particles were dispersed in $n$-heptane in order to avoid particle agglomeration. The experiments were performed on a JEOL 200CX microscope operating at $300 \mathrm{kV}$. For vis-NIR analysis, dilute solutions of the nanocomposite gels without magnetic separation were prepared in deoxygenated water. The spectra were taken in transmission mode using a Shimadzu UV-vis-NIR-3100 instrument, from 200 to $1300 \mathrm{~nm}$ in a $1 \mathrm{~cm}$ quartz cuvette.

The rheological behavior of the nanocomposites was characterized using small-amplitude oscillatory shear measurements using a Bohlin CVO 120 rheometer with parallel plate geometry ( $40 \mathrm{~mm}$ diameter, 1 $\mathrm{mm}$ gap). Immediately after the synthesis, approximately $1.6 \mathrm{~mL}$ of the resultant fluid was transferred to the rheometer sample cell and allowed to gel. The sample was covered with mineral oil to minimize solvent evaporation. The storage modulus $\left(G^{\prime}\right)$ of the gel was measured at $20^{\circ} \mathrm{C}$ as function of the frequency, from 0.1 to $100 \mathrm{rad} / \mathrm{s}$. A strain amplitude of 0.01 was used in all measurements.

The magnetic ac susceptibility was recorded at increasing temperatures (from 5 to $300 \mathrm{~K}$ ) and selected frequencies in the $10-10000$ $\mathrm{Hz}$ range, after an initial cooling from room temperature down to $5 \mathrm{~K}$ in the absence of the field (ZFC procedure). Magnetization was recorded as a function of field (from -90 to $+90 \mathrm{kOe}$ ), at $305 \mathrm{~K}$. All measurements were performed on a PPMS (Quantum Design) magnetometer.

\section{Results}

The ATR-FTIR spectra of the nanocomposite gels (Figure 2a) show a typical carrageenan absorption band around 1225 $\mathrm{cm}^{-1}$ corresponding to the $\mathrm{S}-\mathrm{O}$ asymmetric stretch, the intensity of which is higher for $\lambda$-carrageenan (three sulfates/disaccharide units), compared to $\iota$ - and $\kappa$ - (two and one sulfates/disaccharide unit, respectively). Furthermore, broad absorption bands, due to $\mathrm{C}-\mathrm{O}$ and $\mathrm{C}-\mathrm{OH}$ vibrations characteristic of polysaccharides, are observed in the $1040-1070 \mathrm{~cm}^{-1}$ region. The composites prepared with $\iota$ - and $\kappa$-carrageenan show a clear band at $845-$ $850 \mathrm{~cm}^{-1}$ corresponding the $\alpha(1-3)$-D-galactose $\mathrm{C}-\mathrm{O}-\mathrm{S}$ stretch. $\iota$-Carrageenan also has an additional band around 805 $\mathrm{cm}^{-1}$ due to the sulfate group in the 3,6-anhydrogalactose-2sulfate unit. ${ }^{27,28}$ The $\lambda$-carrageenan sample also gives rise to two bands at 840 and $810 \mathrm{~cm}^{-1}$ attributed to the 2- and 6-sulfate galactose rings, respectively. A broad and intense band centered at around $570 \mathrm{~cm}^{-1}$, corresponding to the $\mathrm{Fe}-\mathrm{O}$ vibration in magnetite, ${ }^{29}$ is observed in the spectra of all composites. The ATR-FTIR spectra are, therefore, consistent with the presence of both carrageenan and iron oxide moieties in all samples.

Figure $2 b$ shows the effect of the addition of both Fe(II) and $\mathrm{Fe}$ (III) cations on the position of the $\mathrm{S}-\mathrm{O}$ stretching band of $\kappa$-carrageenan. In the presence of both ferric and ferrous ions, this band shifts to lower wavenumbers (top spectra), which suggests that the sulfate group is involved in complexation to either $\mathrm{Fe}^{2+}$ or $\mathrm{Fe}^{3+}$ cations. This effect has been observed for 

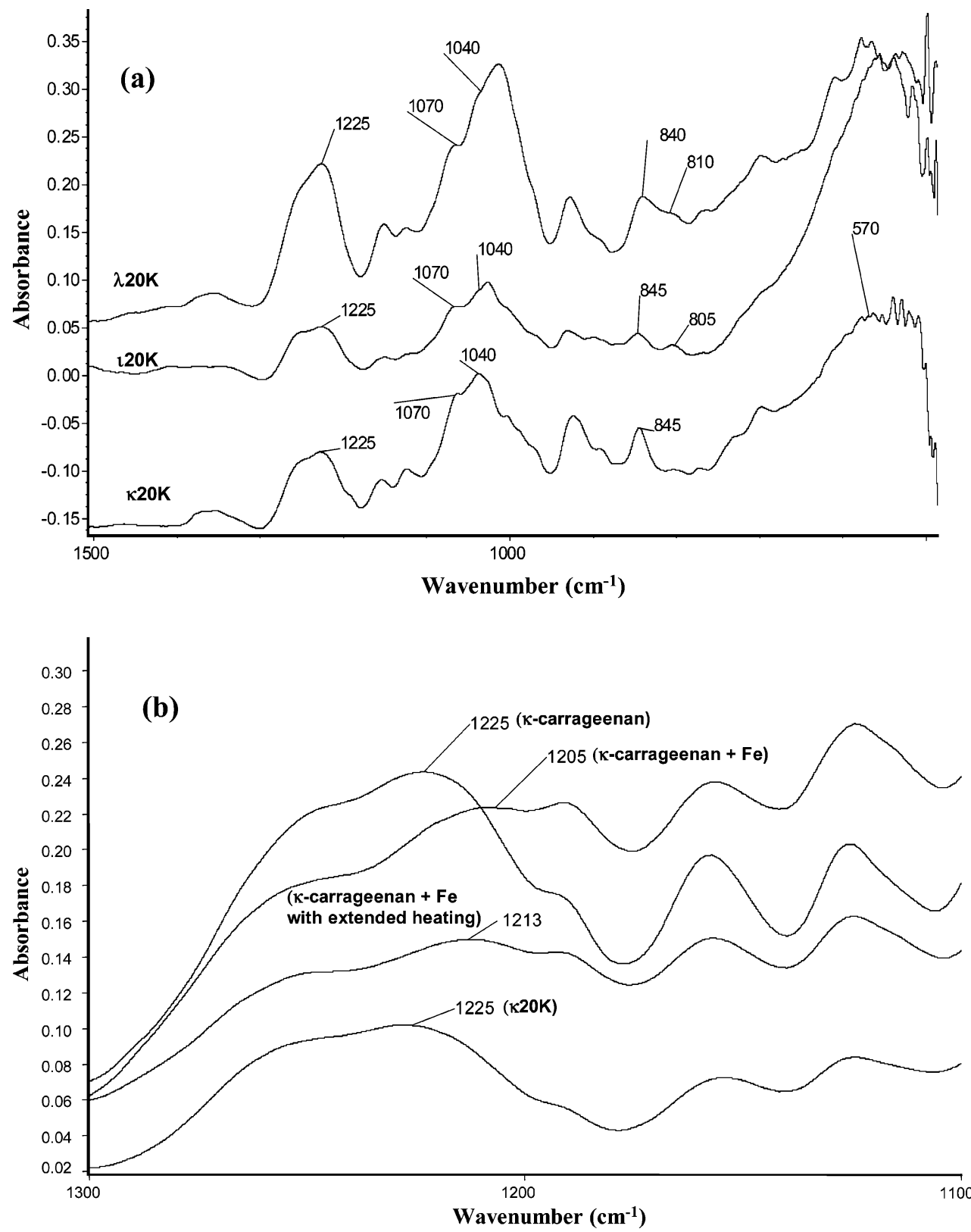

Figure 2. (a) FTIR-ATR spectra of the $\kappa 20 \mathrm{~K}, \iota 20 \mathrm{~K}$, and $\lambda 20 \mathrm{~K}$ nanocomposites. (b) Expansions of the $1100-1300 \mathrm{~cm}^{-1}$ region showing the position of the $\mathrm{S}-\mathrm{O}$ stretch before and after the addition of $\mathrm{Fe}$ to a $\kappa$-carrageenan sol, under standard conditions (top two spectra) and when extended heating at $60^{\circ} \mathrm{C}$ is employed (middle spectrum). The bottom spectrum refers to the sample obtained after addition of hydroxide and subsequent formation of magnetite. Please note the different wavenumber scales in $a$ and $b$.

other sulfated polysaccharides upon complexation. ${ }^{24}$ After formation of magnetite and upon an increase in $\mathrm{pH}$ (bottom spectrum), the $\mathrm{S}-\mathrm{O}$ stretch band shifts to its original position, indicating that the sulfate groups have released Fe. This behavior was also observed here for $\iota$ - and $\lambda$ - carrageenans and is in agreement with the model previously proposed for stabilization of the iron oxide particles in $\kappa$-carrageenan. ${ }^{23}$

Figure $2 \mathrm{~b}$ also shows that, if extended heating (ca. $10 \mathrm{~min}$ ) at $60{ }^{\circ} \mathrm{C}$ is carried out before the addition of alkali for magnetite formation, the $\mathrm{S}-\mathrm{O}$ stretch band shifts to higher wavenumbers, which indicates that a fraction of the carrageenan sulfate moieties are becoming free upon heating. It is also macroscopically noted that, after heating, the resulting gel has become weaker and a shade of orange, which indicates the occurrence of hydrolysis of the cationic iron species and formation of the corresponding iron oxyhydroxides. ${ }^{29}$

The powder XRD difractogram obtained for the $120 \mathrm{~K}$ sample (Figure 3) is representative of those recorded for all samples,

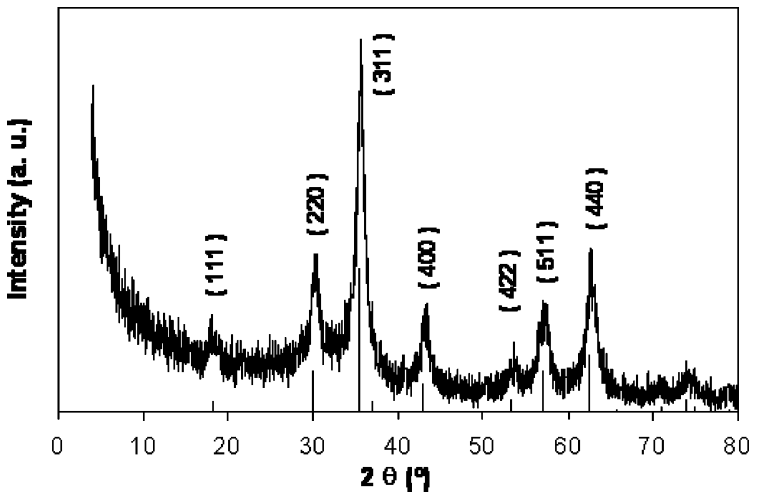

Figure 3. XRD pattern obtained for the $\iota 20 \mathrm{~K}$ sample.

with the exception of the $\lambda$-carrageenan sample, where chemical instability was observed. The powder XRD shown in Figure 3 matches the typical diffraction patterns observed for magnetite, ${ }^{30}$ 

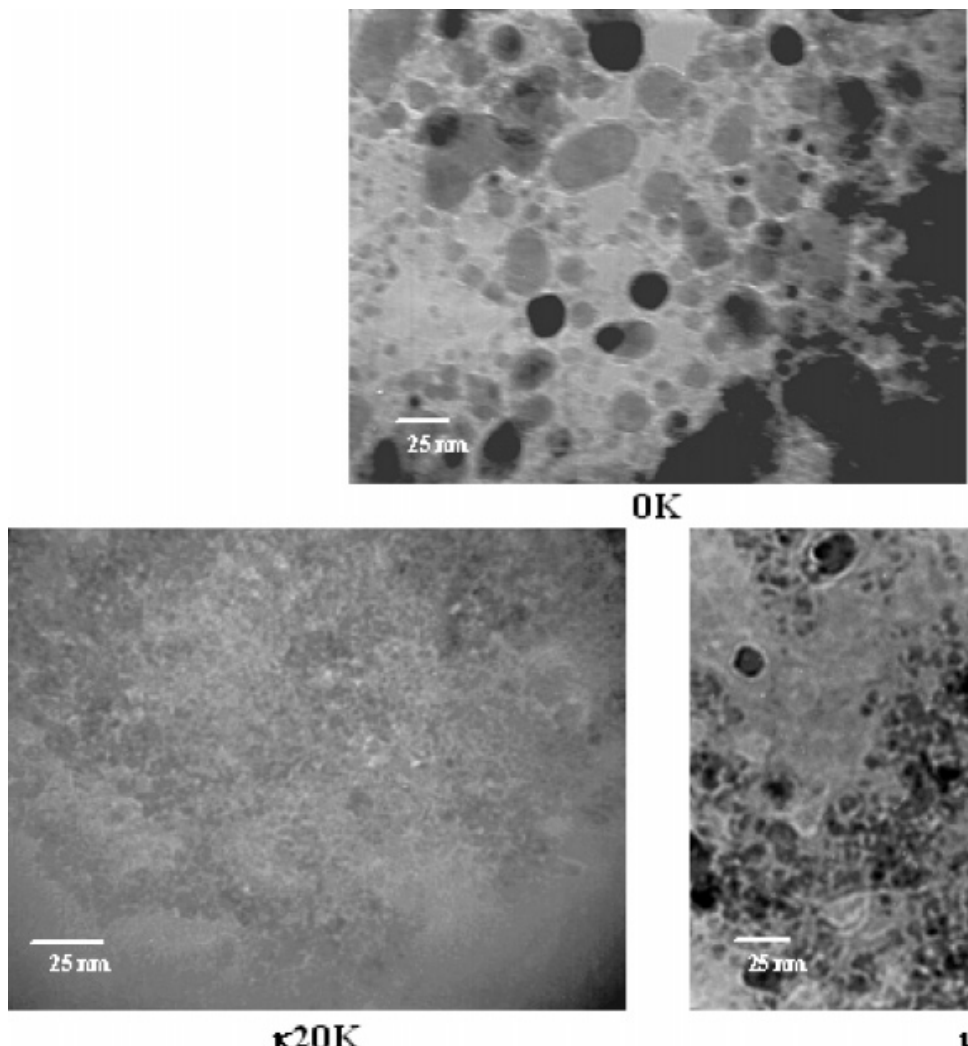

$\mathbf{0 K}$

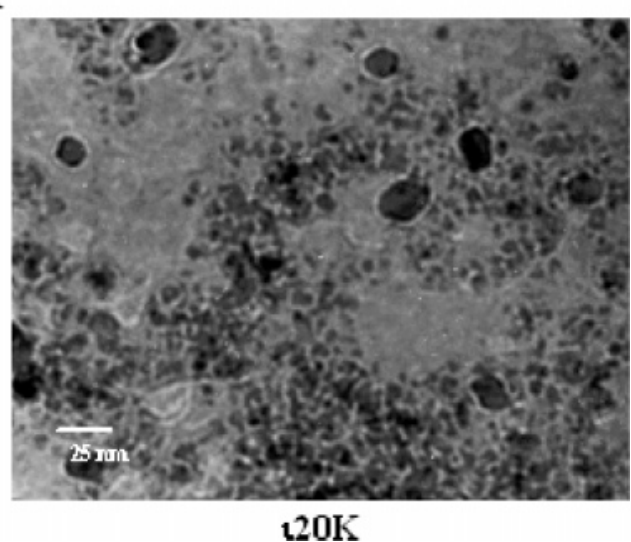

Figure 4. TEM images of magnetic particles obtained for $0 \mathrm{~K}, \kappa 20 \mathrm{~K}$, and $\iota 20 \mathrm{~K}$ samples.

thus confirming that this iron oxide is the main crystalline phase present in the gel, immediately after synthesis. Figure 4 shows the TEM images obtained for the $0 \mathrm{~K}, \kappa 20 \mathrm{~K}$, and $\iota 20 \mathrm{~K}$ magnetite samples. The TEM images show that in the absence of biopolymer, magnetite has particles with higher average sizes compared to those obtained in the presence of carrageenan. For all samples, the average diameter of the magnetite nanoparticles was estimated from the XRD results, using a modified version of Scherrer's equation ${ }^{31-33}$

$$
L=\frac{4}{3} \frac{0.9 \lambda}{\beta_{\mathrm{m}} \cos \theta}
$$

where $\beta_{\mathrm{m}}$ is the peak at half of the maximum intensity (rad) and $\lambda$ the wavelength of $\mathrm{X}$-ray radiation $(1.54178 \AA)$. The average particle size was calculated on the basis of the broadening of all observed reflection peaks. The experimental broadening of each reflection peak fitted by a Gaussian function is expressed as the sum of two main contributions, the crystallite size contribution $\left(\beta_{\mathrm{m}}\right)$ and the instrumental broadening contribution. ${ }^{31}$ Equation 1 is the result of the adaptation of the simple Scherrer formula considering nearly spherical particles $(K=$ $0.9)$ and also taking into account size distribution effects. ${ }^{32,33}$ The results are given in Figure 5 and show, first, that the particles obtained have nanometric dimensions. Second, it is observed that particle size decreases when magnetite is prepared in the presence of carrageenan, and average sizes follow the tendency $\iota$-carrageenan $>\lambda$-carrageenan $>\kappa$-carrageenan. It should be noted that data is not shown for the $\lambda 20 \mathrm{k}$ and $\lambda 40 \mathrm{k}$ samples, as the $\mathrm{Fe}-\mathrm{O}$ particles oxidize before measurements can be recorded. This is in agreement with TEM observations that showed smaller particle sizes for the $\kappa$-carrageenan, compared to $\iota$-carrageenan.

Figure $6 \mathrm{a}$ shows the micrographs of a $\kappa$-carrageenan gel containing iron oxide nanoparticles, taken over a period of 1

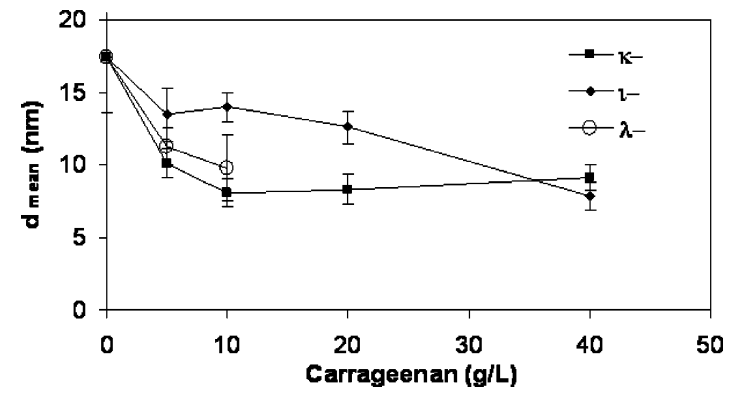

Figure 5. Average particle size evaluated by XRD measurements for $\kappa^{-}, \iota^{-}$, and $\lambda$ - composites ( $\lambda 20 \mathrm{k}$ and $\lambda 40 \mathrm{k}$ data are missing since these systems readily oxidize before measurements can be recorded).

week. In contact with air, the initial black magnetite (left) turns dark brown after about 1 day (middle). An orange-red layer forms next to the air-exposed surface, which, over time, expands to the bottom of the reaction vessel (right). No color changes were observed for magnetite powders prepared in the absence of biopolymer. In fact, it is well-known that at room temperature, magnetite in solution oxidizes very slowly to maghemite, over a period of several months. ${ }^{34}$ In the dry state the process can take several years. ${ }^{29}$ The observations reported here indicate that the presence of carrageenan seems to speed up magnetite oxidation under ambient conditions. Furthermore, the actual rate of magnetite oxidation is seen to depend strongly on the nature of the carrageenan used and its concentration (Figure 6b). For a biopolymer concentration of $20 \mathrm{~g} / \mathrm{L}$, the stability of magnetite prepared in situ follows the trend $\iota$-carrageenan $>\kappa$-carrageenan $>\lambda$-carrageenan. In addition, for $\kappa$ - and $\lambda$-carrageenan, the chemical stability decreases as the biopolymer concentration increases, while, in the presence of $\iota$-carrageenan, this effect seems to be less important and is observed only for higher biopolymer concentrations (e.g., 40 g/L) (Figure 8). If the reacting mixture is heated for longer times (e.g., for 10-15 min), 
a)
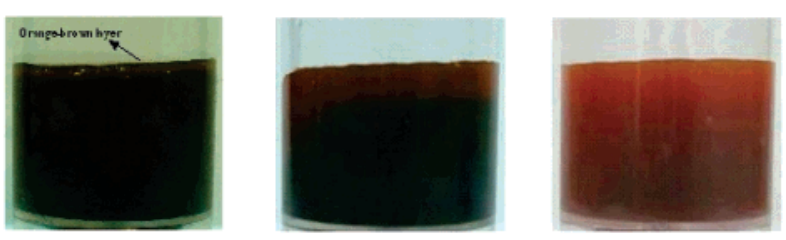

b)

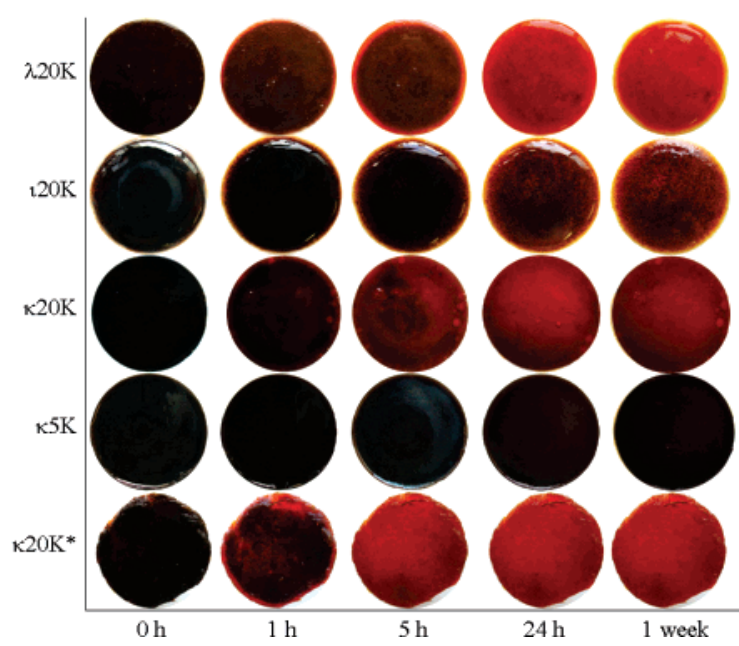

Figure 6. (a) Micrographs of nanocomposite gels of $\kappa$-carrageenan and iron oxide (sample $\kappa 20 \mathrm{~K}$, at room temperature) after $20 \mathrm{~min}$ (left), 1 day (middle), and 1 week (right) (Sample dimensions: $\phi=3 \mathrm{~cm}$, width $=2 \mathrm{~cm}$ ). (b) Color changes observed as a function of time for several nanocomposites gels when exposed to air (sample dimensions: $\phi=3 \mathrm{~cm}$, width $=4 \mathrm{~mm}) .\left(\kappa 20 \mathrm{~K}^{*}: \kappa 20 \mathrm{~K}\right.$ with extended heating (ca. $10 \mathrm{~min}$ ) of the reactants before $\mathrm{pH}$ adjustment).

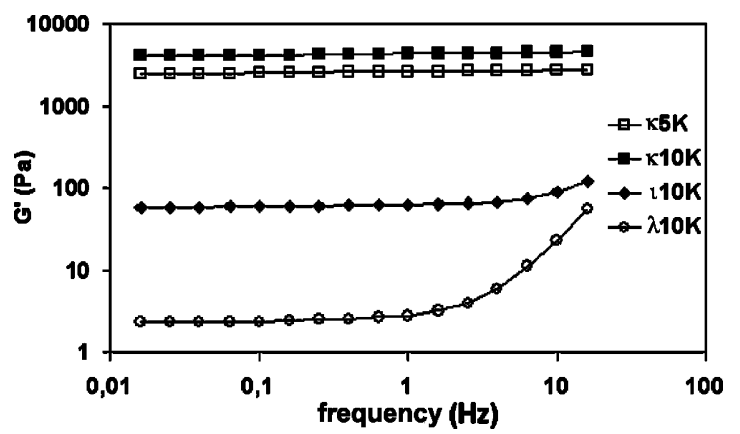

Figure 7. Mechanical spectra of the samples $\kappa 5 \mathrm{~K}, \kappa 10 \mathrm{~K}, \iota 10 \mathrm{~K}$, and $\lambda 10 \mathrm{~K}$ at $20^{\circ} \mathrm{C}$.

before $\mathrm{pH}$ adjustment, the stability of the resultant magnetite also decreases.

Figure 7 illustrates the influence of the nature and concentration of the biopolymer in the rheological properties of the resulting composites. The figure shows the elastic modulus $\left(G^{\prime}\right)$ of the samples as a function of frequency, at $20{ }^{\circ} \mathrm{C}$. It may be seen that the strength of the gel follows the trend $\kappa>\iota>\lambda$. Also, and as expected, increasing biopolymer concentration enhances the strength of the resulting gel. For $\kappa 5 \mathrm{~K}, \kappa 10 \mathrm{~K}$, and $\iota 10 \mathrm{~K}, G^{\prime}$ is almost independent of the frequency, which indicates that the extent of molecular rearrangement is limited, over the frequency scale analyzed, behavior typical of a three-dimensional gel network. On the other hand, for $\lambda 10 \mathrm{~K}$ the higher dependence of the dynamic modulus on frequency is indicative of a weaker structure.

Figure 8 summarizes the visual observations regarding magnetite stability and rheological state for the samples with different carrageenan types and concentrations. Carrageenan

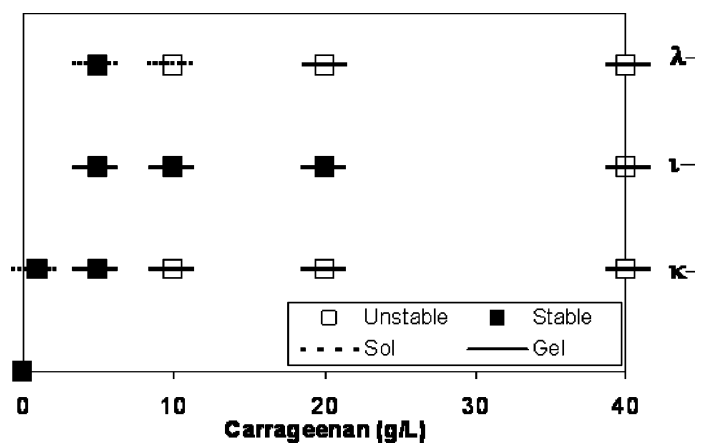

Figure 8. Schematic summary of the results of visual evaluation of (i) magnetite stability after 1 week (full or open squares) and (ii) rheological state (sol or gel, indicated by the dashed or full horizontal lines) of the final nanocomposite (Sample dimensions as in Figure $6 a)$.

concentrations of $\leq 5 \%$ do not seem to affect magnetite chemical stability significantly. However, at higher concentrations, $l$-carrageenan samples show chemical stability up to a polymer concentration of $20 \mathrm{~g} / \mathrm{L}$, while the remaining magnetite particles become unstable at a carrageenan concentration of $10 \mathrm{~g} / \mathrm{L}$ and thereafter. This suggests that increasing the gel strength (expected for higher concentrations) leads to a decrease of the stability of the resultant magnetic particles. This may relate to the already noted tendency for lower average particle sizes with increasing concentration (Figure 5), with smaller particles undergoing faster oxidation. ${ }^{34,35}$ The effect of small changes in gel strength, obtained by varying the specific cation present $\left(\mathrm{Na}^{+}, \mathrm{K}^{+}\right.$, or $\left.\mathrm{Cs}^{+}\right)$, was also investigated, but results showed that chemical stability did not depend on the nature of the counterion. Furthermore, the results represented in Figure 8 also suggest that chemical stability does not seem to depend in a straightforward way on whether the sample is a sol or a gel, since the sol to gel changes do not seem to be accompanied by a change in chemical stability.

In an attempt to identify the form or forms of oxidized magnetite obtained, monitoring by vis-NIR spectroscopy was carried out. As only magnetite and wustite $(\mathrm{FeO})$ show finite absorption in the NIR region, ${ }^{34}$ it should be possible to follow the disappearance of magnetite via the loss of near-IR absorbance. Figure 9 shows the evolution of the vis-NIR spectra of dilute solutions of the $\kappa 20 \mathrm{~K}$ gel exposed to air for different lengths of time. The NIR absorbance is seen to decrease with time, indicating that magnetite is being transformed into an iron oxide species that does not absorb in the NIR region. The inset shows the whole vis-NIR spectrum, which is in agreement with the typical spectrum found for several iron oxides, including magnetite. ${ }^{23,34}$ Although these spectroscopic results are consistent with magnetite nanoparticles undergoing oxidation, in the presence of carrageenan and at ambient conditions, they do not allow conclusions to be drawn as to the nature of the final iron oxide phase present in the gel.

The Mössbauer spectrum of the nanoparticles magnetically separated from the $\iota 20 \mathrm{~K}$ sample (Figure 10a) was obtained to try and determine the nature of the resulting iron oxides. The presence of magnetite nanoparticles in the gel, at $t=0$, was indicated by two sextets corresponding to tetrahedral and octahedral coordination of the iron sites in the magnetite. In addition, the spectrum in Figure 10a also shows one doublet, which indicates the presence of a nonmagnetic ferric oxide containing $\mathrm{Fe}^{3+}$ centers. This same species was also present in the lower concentration samples of $\kappa$ - and $\lambda$-carrageenan samples, for which apparent chemical stability was observed, and it may indicate that the presence of the nonmagnetic iron 


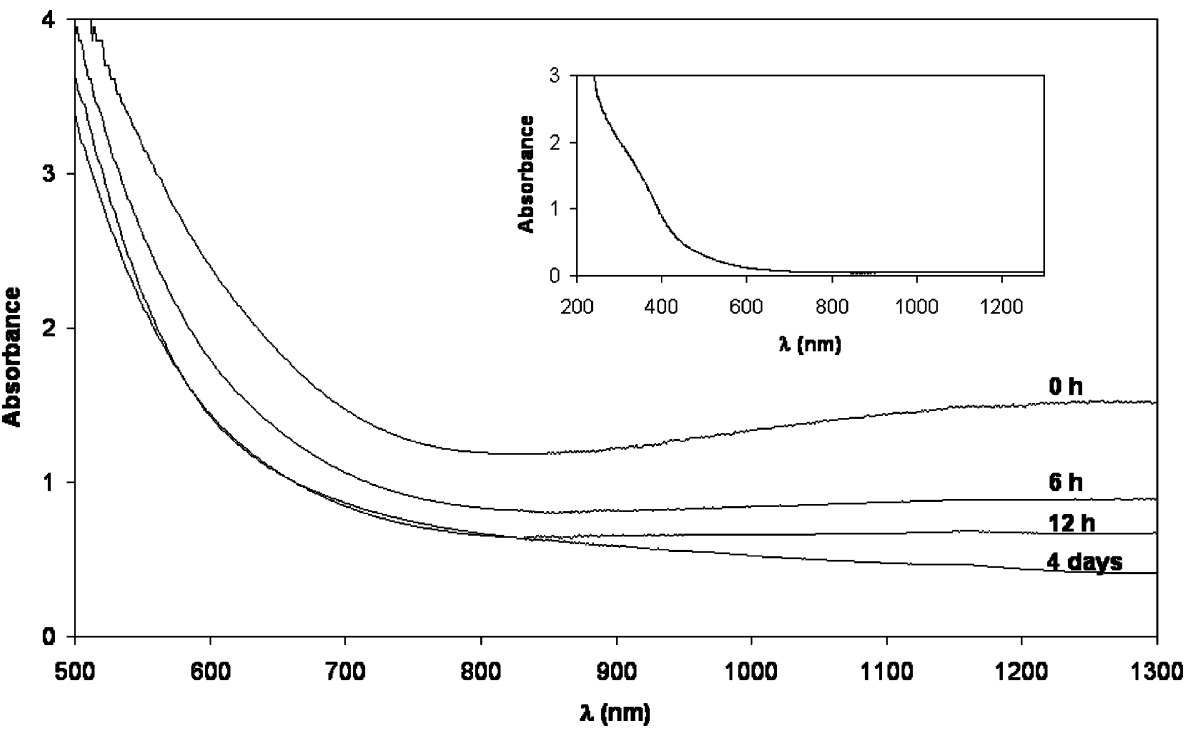

Figure 9. Vis-NIR region of the absorbance spectra for dilute solutions of $\kappa 20 \mathrm{~K}(0.3 \mathrm{~g} \mathrm{gel} / \mathrm{mL})$ exposed to air for different times at room temperature. The inset shows the whole vis-NIR spectrum for a further diluted solution of $\kappa 20 \mathrm{~K}$ before oxidation has occurred.

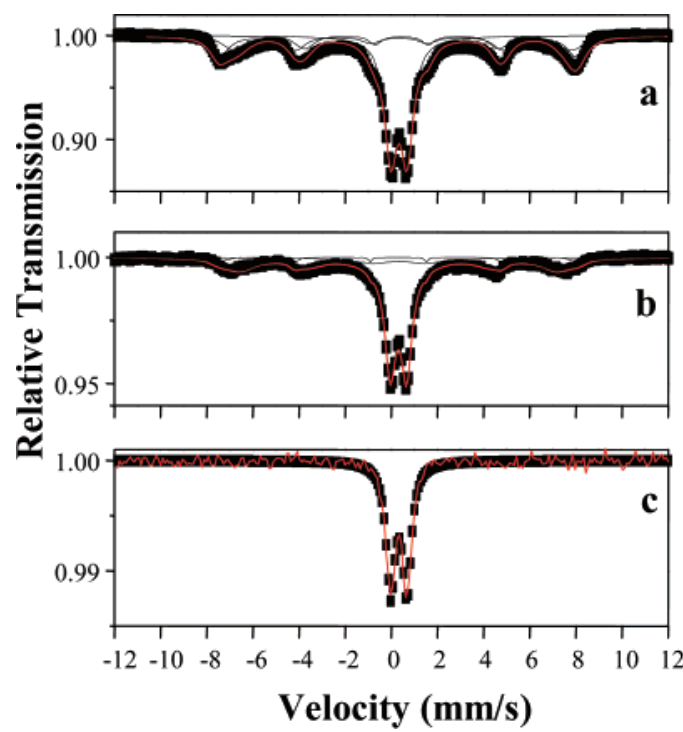

Figure 10. Mössbauer spectra obtained for the $\iota 20 \mathrm{~K}$ sample (a) after magnetic separation of the nanoparticles immediately after synthesis, (b) sample as in part a after exposure to air for a period of 4 months and (c) whole gel sample (i.e., with no previous separation of the magnetic nanoparticles) after exposure to air for 4 months. Red lines represent the theoretical fitting of the experimental data.

species results from partial oxidation of the sample. After exposure for a period of 4 months, the Mössbauer spectrum of the $220 \mathrm{~K}$ sample (Figure 10b) shows clearly the decrease of magnetite content, probably due to its oxidation. The Mössbauer spectrum of the oxidized gel (with no previous magnetic separation of the nanoparticles) after the same period of time (4 months) shows only one doublet, which indicates that the nonmagnetic iron(III) oxide (Figure 10c) is the only inorganic phase present in the gel. Similar results have been obtained for the oxidized $\kappa 20 \mathrm{~K}$ and $\lambda 20 \mathrm{~K}$ nanocomposite gels, no XRD peaks typical of hematite or maghemite having been found for any of the samples data recorded.

Magnetic measurements were performed for the sample $(\kappa 5 \mathrm{~K})$ showing the highest stability in relation to oxidation. At $T=$ $305 \mathrm{~K}$, the magnetite nanoparticles have zero remanence and coercive field ( $\mathrm{Hc}=0 \pm 10 \mathrm{Oe}$ ) (Figure 11). As a first approximation, the nanoparticles magnetic moment $\mu$ can be estimated by using the Langevin law $^{36}$ to model the $M(H)$ curve

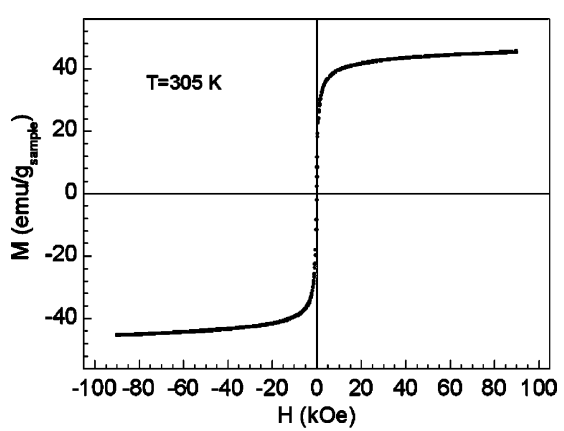

Figure 11. Magnetization of sample $\kappa 5 \mathrm{~K}$ as function of magnetic field at $305 \mathrm{~K}$.

(in this approximation we consider negligible the $\mu$ distribution, dipolar interactions, and anisotropy): $\mu \approx 8000 \mu_{B}$. Assuming that the sample is mainly composed of spherical magnetite particles, the saturation magnetization of bulk magnetite at room temperature is $\sim 82 \mathrm{emu} / \mathrm{g} \mathrm{Fe}_{3} \mathrm{O}_{4}$, and the density of magnetite is $\sim 5 \mathrm{~g} / \mathrm{cm}^{3}$, the nanoparticles radius can be estimated as 10 $\mathrm{nm}$. This value agrees with that found on the basis of susceptibility measurements (Figure S1 in Supporting Information) and is on the order of that found by XRD. Also, the inphase $\left(\chi^{\prime}\right)$ and out-of-phase $\left(\chi^{\prime \prime}\right)$ ac susceptibility measurements performed in the nanocomposite sample showed a frequency (f) dependency for the temperature range 5-300 K (Figure S1 in Supporting Information), showing that the magnetite nanoparticles are superparamagnetic, being blocked in the studied temperature and frequency range.

\section{Discussion}

FTIR and XRD results suggest that magnetite is the main crystalline phase formed during the synthesis described here. However magnetite and maghemite possess similar XRD patterns; thus, unequivocal evidence of the presence of magnetite in the sample was obtained by Mössbauer spectroscopy (Figure 10a).

FTIR results have shown that the synthesis of magnetite nanoparticles in the presence of carrageenans seems to involve the coordination of ferric (and ferrous) ions to the sulfate group of the biopolymer, in a first stage, promoting the physical crosslinking of the biopolymer chains and formation of a microgel 
at low pH. Previous FTIR studies have shown similar results for other sulfated polysaccharides, ${ }^{24}$ and thermogravimetric and differential scanning calorimetric measurements have shown that $\kappa$ - and $\iota$-carrageenans may also coordinate to ferric ions via their hydroxyl groups. ${ }^{37-39}$ After the $\mathrm{pH}$ increase to form iron oxide particles, FTIR spectra show that at least part of the sulfate groups are released. This is consistent with the previous data ${ }^{23}$ suggesting that, after an initial sulfate/iron chelating step and upon increasing the $\mathrm{pH}$, self-capped iron oxide nanoparticles are formed involving biopolymer molecules. Within these structures, iron ions migrate to the particle nucleation sites, leaving a number of unbound sulfates behind. However, some sulfate moieties should still remain coordinated to the nanoparticle surfaces, thus accounting for the high colloidal stability of some of these systems.

The results described here indicate that the presence of carrageenan alone has a significant effect on the magnetite particle size. Nanoparticles prepared in the presence of carrageenan show lower average sizes compared to those prepared in its absence, and in general, particle size decreases with increasing biopolymer concentration (Figure 5). In addition, the nature of the carrageenan plays an important role, with $l$-carrageenan nanoparticles showing higher average sizes, compared to $\lambda$ - and $\kappa$-carrageenan. This may be due to the stabilization of hydrolyzed iron species by the particular sulfate charge distribution of $\kappa$-carrageenan. Therefore, for a certain iron load, this biopolymer seems to act better as a template for magnetite particle formation. It has been previously reported that $\kappa$-carrageenan stabilizes iron oxyhydroxides at higher Fe/polysaccharide ratio than either $\iota$ - or $\lambda$-carrageenan. ${ }^{23}$ Also, it has been observed that iron stabilization decreases as the Fe/polysaccharide ratio increases, which is consistent with the higher average particle sizes found here at lower biopolymer concentrations. The addition of ferric ions to $\iota$-carrageenan leads to the formation of a heterogeneous gel, with harder orange zones in a yellow soft matrix, while homogeneous and transparent yellow gels were obtained for $\kappa$ - and $\lambda$-carrageenan. This may be an indication that $\iota$-carrageenan does not stabilize (or bind) all the added iron, which could explain the higher average particle size and also the wider particle size distribution obtained when this biopolymer is used.

The oxidation of magnetite in the presence of $\kappa$-carrageenan at room temperature has previously been reported, but no explanation was proposed. ${ }^{23}$ Our results show that the stability to oxidation of the in situ generated magnetite, under ambient conditions, depends strongly on the type of carrageenan used. $\iota$-Carrageenan-coated particles exhibit the lowest oxidation rate (highest stability), which may be a direct consequence of the highest average particle size found for this system. Indeed, the observed tendency for average particle size to decrease, as carrageenan concentration increases, is consistent with the observed faster oxidation at higher concentrations. This is in agreement with the literature, ${ }^{34,35}$ where the oxidation of magnetite is suggested to occur through the outward diffusion of ferrous cations; in small crystals, the diffusion length is short and therefore the oxidation process is fast.

However, the relative stability to oxidation of the magnetite in different carrageenan systems $(\iota>\kappa>\lambda)$ does not follow the trend in particle size $(\iota>\kappa>\lambda) . \kappa$ - And $\lambda$-carrageenan show opposite trends to those expected solely on the basis of particle size. We suggest, therefore, that the effect of the biopolymer on stability to oxidation of in situ generated magnetite nanoparticles results from a balance between two factors: (i) average particle size and (ii) the rheological state (sol or gel) of the resulting nanocomposite. In the case of $\kappa$-carrageenan, and in spite of the smaller particle size, chemical stability is probably enhanced due to the stronger gels formed by this polysaccharide, compared to $\lambda$-carrageenan. Simple rheological measurements have confirmed the expected relative strength of the gellified samples (Figure 7). Thus, the biopolymer not only acts as a template controlling the iron oxide nanoparticles growth but, by forming a gel of varying strength, may also determine the oxygen diffusion rate within the material and hence affect oxidation rates. For extended heating time before $\mathrm{pH}$ adjustment, the resulting magnetite is less stable, which is consistent with the weaker nature of the resulting gel, which should promote higher oxygen diffusion. However, heating may also change the morphology of the magnetite formed due to the formation of iron oxyhydroxydes, resulting in reduced chemical stability. However, this could not be confirmed by particle size measurements, as magnetite oxidation in the heated sample occurred too fast.

The balance of the two effects is exquisitely dependent on the nature of the polysaccharide. Indeed, $\iota$-carrageenan-coated particles exhibit considerable stability, due to their large particle size and intermediate gel strength; $\lambda$-carrageenan-coated particles show the lowest chemical stability, due to the comcomitant effects of reduced particle size and low gel strength; and $\kappa$-carrageenan-coated particles show a balanced result, due to the low particle size and higher gel strength (the latter effect compensating the former) in this system. $\kappa$-And $\iota$-carrageenan composites are, therefore, the best candidates for future applications, due to their enhanced chemical stability toward oxidation. For $\kappa$-carrageenan, a good balance between particle size and chemical stability seems to be achieved at concentrations of about $5 \mathrm{~g} / \mathrm{L}$, for which a gel containing more stable magnetic nanoparticles is obtained. However, even for this biopolymer concentration, the nanocomposite gel turns dark brown after about 3-4 weeks, which indicates that magnetite oxidation does eventually occur. For $\iota$-carrageenan at the same concentration $(5 \mathrm{~g} / \mathrm{L})$, the particles are slightly larger (average diameter just under $15 \mathrm{~nm}$, compared to $10 \mathrm{~nm}$ for $\kappa$-carrageenan) and stability is extended for a further few weeks.

Although hematite $\left(\alpha-\mathrm{Fe}_{2} \mathrm{O}_{3}\right)$ has been shown to be the thermodynamically favored phase resulting from the oxidation of magnetite to maghemite, ${ }^{29}$ our results (Figure 10) do not confirm the presence of any of these magnetic crystalline phases in the final oxidized gels. At this stage, we can only note that the oxidized solid phase consists mainly of small nanoclusters of an nonmagnetic iron(III) oxide that form at the expense of magnetite nanoparticles. This conversion seems to be faster for the unseparated sample, in which the nanoparticles remain in the polysaccharide solution (Figure 10c), compared to the nanocomposite after separation from solution (Figure 10b). However, we have observed that in the unseparated samples the nanocomposite particles are still attracted to an adjacent magnet. These apparently contradictory results may indicate that, over the whole nanocomposite sample, magnetite is not totally oxidized and remains as very small magnetic cores (less than $10 \%$, which is the minimum sensitivity of the Mossbauier instrument). This would explain the fact that the powder XRD did not show evidence for any known iron (III) oxide phases.

The analysis of the magnetic properties of the sample showing the best balance between particle size and chemical stability $(\kappa 5 \mathrm{~K})$ revealed that the magnetite nanoparticles are superparamagnetic (Figure 11). This result is in agreement with reported work on the superparamagnetic behavior of magnetite within the particle size range of $8-30 \mathrm{~nm}^{40}$ 


\section{Conclusions}

Magnetite nanoparticles were successfully prepared by an in situ coprecipitation method using the polysaccharide carrageenan as a biopolymeric matrix. The carrageenan is suggested to act as a self-assembled nanoreactor for magnetite formation, resulting in polymer-encapsulated magnetite nanoparticles. The formation of magnetite as the main magnetic phase was confirmed by Mössbauer spectroscopy. It is well-known that the synthesis of nanoparticles in the presence of soft templates, such as biopolymers, usually present less morphological control compared to lyothermal methods involving coordinating solvents. However, it has been observed here that both the morphology, namely particle size, and stability toward oxidation of the in situ generated magnetite strongly depend on the type and concentration of carrageenan used. Considering that increasing biopolymer concentration results in smaller nanoparticles and stronger gels and that magnetite oxidation follows a sizedependent diffusion mechanism, it is suggested that small particle sizes accelerate oxidation while strong gels have the opposite effect, as oxygen diffusion within the gel becomes more difficult.

The highest stability toward oxidation was observed for the $\iota$-carrageenan nanocomposite, a system which is characterized by an average particle diameter observed of just under $15 \mathrm{~nm}$, at $5 \mathrm{~g} / \mathrm{L}$. At the same concentration, the $\kappa$-carrageenan nanocomposite follows in terms of chemical stability and is characterized by an average particle diameter of about $10 \mathrm{~nm}$. For this system, an optimum concentration of $5 \mathrm{~g} / \mathrm{L}$ was found, giving a gel that contains relatively stable magnetite nanoparticles. Nevertheless, it should be noted that long-term $(>1$ month) oxidation still occurs in all systems, and therefore, susceptibility to oxidation must be considered when exploring practical applications of magnetite encapsulated in carrageenan.

Acknowledgment. The authors thank Dr. Nuno João Silva (University of Zaragoza, Spain) and Ms. Penka Girginova (University of Aveiro, Portugal) for carrying out the measurements of magnetic properties and for the useful analysis and interpretation of resulting data. We also thank Dr. J. Lopes da Silva (University of Aveiro, Portugal) for guidance in the rheological studies of the samples under investigation. A.L.D. da S. thanks CICECO-Centro de Investigação em Materiais Cerâmicos e Compósitos (University of Aveiro) for funding this work.

Supporting Information Available. Magnetic ac susceptibility of the sample $\kappa 5 \mathrm{~K}$ as a function of temperature for different ac field frequencies (Figure S1). This material is available free of charge via the Internet at http://pubs.acs.org.

\section{References and Notes}

(1) Rabelo, D.; Lima, E. C. D.; Reis, A. C.; Nunes, W. C.; Novak, M. A.; Garg, V. K.; Oliveira, A. C.; Morais, P. C. Nano. Lett. 2001, 1, $105-108$.

(2) Qu, S.; Yang, H.; Ren, D.; Kan, S.; Zou, G.; Li, D.; Li, M. J. Colloid Interface Sci. 1999, 215, 190-192.

(3) Si, S.; Kotal, A.; Mandal, T. K.; Giri, S.; Nakamura, H.; Kohara, T. Chem. Mater. 2004, 16, 3489-3496.

(4) Ditsch, A.; Laibinis, P. E.; Wang, D. I. C.; Hatton, T. A. Langmuir 2005, 21, 6006-6018.
(5) Liu, T. -Y.; Hu, S. -H.; Liu, T. -Y.; Liu, D. -M.; Chen, S. -Y. Langmuir 2006, 22, 5974-5978.

(6) Cocker, T. M.; Fee, C. J.; Evans, R. A. Biotechnol. Bioeng. 1997, $53,79-87$

(7) Lin, H.; Watanabe, Y.; Kimura, M.; Hanabusa, K.; Shirai, H. J. Appl. Polym. Sci. 2003, 87, 1239-1247.

(8) Breulmann, M.; Colfen, H.; Hentze, H. -P.; Antonietti, M.; Walsh, D.; Mann, S. Adv. Mater. 1998, 10, 237-241.

(9) Pardoe, H.; Clura-Anusorn, W.; St. Pierre, T. G.; Dobson, J. J. Magn. Magn. Mater. 2001, 225, 41-46.

(10) Honda, H.; Kawaba, A.; Shinkai, M.; Kobayashi, T. J. Ferment. Bioeng. 1998, 86, 191-196.

(11) Gupta, A. K.; Gupta, M. Biomaterials 2005, 26, 3995-4021.

(12) Kim, D. K.; Voit, W.; Zapka, W.; Bjelke, B.; Muhammed, M.; Rao, K. V. Mat. Res. Soc. Symp. Proc. 2002, 676, Y.8.32.1-Y.8.32.6.

(13) Ito, A.; Shinkai, M.; Honda, H.; Kobayashi, T. J. Biosci. Bioeng. 2005, 100, 1-11.

(14) LaConte, L.; Nitin, N.; Bao, G. Nanotoday 2005, 8, 32-38.

(15) Tartaj, P.; Morales, M. P.; González-Carreño, T.; VeintemillasVerdaguer, S.; Serna, C. J. J. Magn. Magn. Mater. 2000, 290-291, $28-34$.

(16) Gamarra, L. F.; Brito, G. E. S.; Pontuschka, W. M.; Amaro, E.; Parma, A. H. C.; Goya, G. F. J. Magn. Magn. Mater. 2005, 289, 439-441

(17) Cao, Z.; Zhou, S.; Liu, J.; Song, X. Chin. Ger. J. Clin. Oncol. 2005, 4, $183-186$.

(18) Duan, H. -L.; Shen, Z. -Q.; Wang, X. -W.; Chao, F .-H.; Li, J. -W World J. Gastroenterol. 2005, 11, 3660-3664.

(19) Bonacchi, D.; Caneschi, A.; Gatteschi, D.; Sangregorio, C.; Sessoli, R.; Falqui, A. J. Phys. Chem. Solids 2004, 65, 719-722.

(20) Shen, F.; Poncet-Legrand, C.; Somers, S.; Slade, A.; Yip, C.; Duft, A. M.; Winnik, F. M.; Chang, P. L. Biotechnol. Bioeng. 2003, 83, $282-292$

(21) Llanes, F.; Diaz, C.; Ryan, H.; Marchessault, R. H. Int. J. Polym Mater. 2002, 51, 537-545.

(22) Kim, D. K.; Mikhaylova, M.; Wang, F. H.; Kehr, J.; Bjelke, B.; Zhang, Y.; Tsakalakos, T.; Muhammed, M. Chem. Mater. 2003, 15, 4343-4351.

(23) Jones, F.; Colfen, H.; Antonietti, M. Colloid Polym. Sci. 2000, 278 , 491-501.

(24) Sipos, P.; St. Pierre, T. G.; Tombacz, E.; Webb, J. J. Inorg. Biochem. 1995, 58, 129-138.

(25) Jones, F.; Colfen, H.; Antonietti, M. Biomacromolecules 2000, 1, $556-563$.

(26) Piculell, L. In Food Polysaccharides and Their Applications; Stephen, A. M., Ed.; Marcel Dekker: New York, 1995; pp 205-215.

(27) Prado-Fernández, J.; Rodríguez-Vázquez, J. A.; Tojo, E.; Andrade, J. M. Anal. Chim. Acta 2003, 480, 23-37.

(28) Pereira, L.; Sousa, A.; Coelho, H.; Amado, A. M; Ribeiro-Claro, P. J. A. Biomol. Eng. 2003, 20, 223-228.

(29) Cornell, R. M.; Schwertmann, U. In The Iron Oxides-Structure, Properties, Reaction, Occurrences and Uses; Wiley-VCH: Weinheim, 2003; p 402.

(30) Joint Committee for Powder Diffraction Studies, JCPDS, Card No. 19-0629.

(31) Hammond, C. In The Basics of Crystallography and Diffraction; Oxford Science Publications: Oxford, 1997; pp 145-148.

(32) Natter, H.; Schmelzer, M.; Löffler, M. -S.; Krill, C. E.; Fitch, A.; Hempelmann, R. J. Phys. Chem. B 2000, 104, 2467-2476.

(33) Borchert, H.; Shevchenko, E. V.; Robert, A.; Mekis, I.; Kornowsk, A.; Grübel, G.; Weller, H. Langmuir 2005, 21, 1931-1936.

(34) Tang, J.; Myers, M.; Bosnick, K. A.; Brus, L. E. J. Phys. Chem. B 2003, 107, 7501-7506.

(35) Murad, E.; Schwertmann, U. Clay Clay Miner. 1993, 41, 111-113.

(36) Elmore, W. C. Phys. Rev. 1938, 54, 1092-1095.

(37) Ciesielski, W. J. Food Agr. Environ. 2004, 2, 17-25.

(38) Ciesielski, W. J. Food Agr. Environ. 2004, 2, 26-32.

(39) Ciesielski, W. J. Food Agr. Environ. 2004, 2, 33-38.

(40) Jeong, U.; Teng, X.; Wang, Y.; Yang, H.; Xia, Y. Adv. Mater. 2007, $19,33-60$.

BM070096Q 